\title{
Example of Using Pythia8 to Decay Resonances in Geant4
}

\author{
Julia Yarba, Fermilab \\ $26^{\text {th }}$ Geant4 Collaboration Meeting \\ Sept 16, 2021
}

FERMILAB-SLIDES-21-103-SCD 


\section{examples/extended/eventgenerator/py8decayer (I)}

- Addresses requests from the community

- Initially, interest from the local Fermilab projects

- Per discussions in the Geant4 Hadronic group with regards to decays of B's, etc.; also, desire from various users (incl. LHC's) on adding such Pythia8-based example was communicated to us

- Included with Geant4 distribution since 11.7.r06 (11.0.beta)

- Demonstrates how to use Pythia8-based decay features with those resonances in Geant4 where decay tables are not implemented by default

- Shows how to replace existing Geant4 decay tables to such resonances as tau+/- or B+/- with the Pythia8-based ones

- Draws inspiration in existing similar example based on Pythia6 


\section{examples/extended/eventgenerator/py8decayer (II)}

- Building this example is optional; activated by setting PYTHIA8 environment variable to point to the Pythia8 area

- Step-by-step ("cookbook style") README is provided

- In case Pythia8 is not centrally installed, the README also provides step-by-step instruction for building it (only a minimal configuration of Pythia8 is needed in this case)

- Source code is annotated with comments

- Minimalistic implementation so that not to distract attention from the main feature: use of Pythia8 as "external decayer":

- Simplistic geometry, single particle gun, and the Pythia8 decay physics module which is registered with FTFP_BERT

- Not even UI-based job configuration (but can be added)

- As of right now, operates on SerialOnly RunManager 


\section{examples/extended/eventgenerator/py8decayer (III)}

- A few technicalities to point out:

- Example shows how to largely silence Pythia8 (incl. banner), and to allow the output of only some desired information; however, if users want full Pythia8 information to be printed out, they can skip silencing it (see comments in the code)

- By default Pythia8 decays resonances all the way through, until there are only stable particles in the tree; however, in certain cases users may want to pass some of the decay products, e.g. pi0's, undecayed to Geant4 and have Geant4 treat them; this example shows to to do so

- When transferring back and forth between Geant4 and Pythia8, and again, we use Geant4 Particle Data Table (PDT) - still need to double check for consistency of PDT's in these two packages (hopefully, there will be no concerns)

- Feedback is most welcome, and will certainly help to further improve this example! 